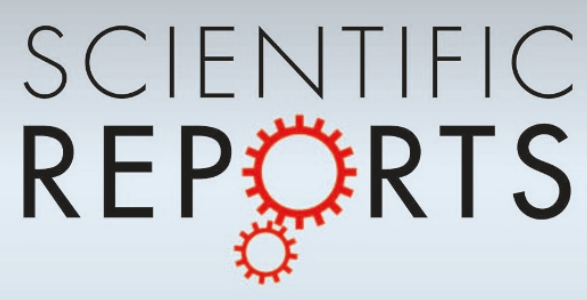

\title{
open Magnetic Vortex Based Transistor Operations
}

SUBJECT AREAS:

MAGNETIC DEVICES

METAMATERIALS

MAGNETIC PROPERTIES AND MATERIALS

COMPUTATIONAL METHODS

Received

28 August 2013

Accepted

30 January 2014

Published

17 February 2014

Correspondence and requests for materials should be addressed to

S.B. (saswati@bose. res.in) or A.B. (abarman@bose.res.

in)

\author{
D. Kumar, S. Barman \& A. Barman
}

Thematic Unit of Excellence on Nanodevice Technology, Department of Condensed Matter Physics and Material Sciences, S. N. Bose National Centre for Basic Sciences, Block JD, Sector III, Salt Lake, Kolkata 700 098, India.

Transistors constitute the backbone of modern day electronics. Since their advent, researchers have been seeking ways to make smaller and more efficient transistors. Here, we demonstrate a sustained amplification of magnetic vortex core gyration in coupled two and three vortices by controlling their relative core polarities. This amplification is mediated by a cascade of antivortex solitons travelling through the dynamic stray field. We further demonstrated that the amplification can be controlled by switching the polarity of the middle vortex in a three vortex sequence and the gain can be controlled by the input signal amplitude. An attempt to show fan-out operation yielded gain for one of the symmetrically placed branches which can be reversed by switching the core polarity of all the vortices in the network. The above observations promote the magnetic vortices as suitable candidates to work as stable bipolar junction transistors (BJT).

$\top$ here has been a revolution in the study of inhomogeneous and non-trivial magnetic nano-structures such as magnetic vortices and antivortices due to their suggested applications in magnetic data storage, magnetic random access memory ${ }^{1-4}$, magnetic logic ${ }^{5}$ and information processing devices ${ }^{5}$. The presence of rotational asymmetry makes them very attractive candidates for studying the interaction between the local magnetization and externally applied magnetic fields or spin polarized currents ${ }^{6-16}$. Magnetization can align itself parallel to the edges of a thin ferromagnetic nano-disk in order to reduce the surface charges. This gives rise to the clockwise (CW) or counter-clockwise (CCW) chirality of the vortex. The core of this vortex, which is a few nanometres in diameter, gets pushed out $(p=1)$ or into $(p=-1)$ the nano-disk's plane as the exchange interaction favours a parallel arrangement. This is defined as the polarity of the vortex.

Magnetic vortices can be brought to gyration by the application of magnetic fields or spin polarized currents ${ }^{1,16-20}$. In addition to the external forces, the moving vortex core experiences another internal force arising from the demagnetizing field of the non-equilibrium magnetization pattern. This force is perpendicular to the vortex core's velocity and leads to its gyrotropic motion. For large amplitude excitation, vortex core switching occurs along with the creation and annihilation of new vortex and antivortex pair ${ }^{21,22}$. For small amplitude excitation, vortex core motion remains in the linear regime ${ }^{23}$.

The magnetization dynamics resulting in the gyrotropic vortex core motion is described by the LandauLifshitz-Gilbert equation ${ }^{24,25}$. In the linear regime, the vortex core equation of motion can be derived from the Thiele's equation ${ }^{23}$. The CW or CCW sense of vortex core gyration direction is solely controlled by its polarity. In the linear regime, vortex core can be described by a harmonic oscillator mode ${ }^{26}$. Consequently, magnetostatically coupled vortex gyration can be considered as coupled oscillators. Therefore, one expects mutual energy transfer and a consistent phase relation between the gyrating vortices ${ }^{27,28}$. Logic operations based on magnetic vortex state networks have been demonstrated experimentally via the vortex gyration mediated information signal transfer mechanism ${ }^{29}$.

Subsequently, the mutual transfer of energy between magnetostatically coupled vortices, where one of the vortices is locally excited, is extremely important for microwave communication and logic operations. In this regard, the parameters like the signal transport rate and efficiency are the key factors in determining the device performance. Vortex gyration transfer rate and energy attenuation coefficients have been calculated by analytical method and micromagnetic simulations ${ }^{30}$. Stimulated vortex gyration based energy transfer between spatially separated dipolar coupled magnetic disks has been observed by time resolved soft $\mathrm{x}$-ray microscopy ${ }^{13}$. The rate of energy transfer is found to be determined by the frequency splitting caused by the dipolar interaction between the vortices $^{29}$.

This energy transfer efficiency may depend on several factors such as the frequency of the exciting field pulse as compared to the gyration frequency of the vortex core, the amplitude and nature of the exciting pulse, the distance between the vortices and their relative polarity. Until recently ${ }^{13}$, the energy transfer efficiency was found to be well 
below $100 \%{ }^{27,28}$ and no amplification has been reported thus far. It has also been reported that the interaction strength between coupled vortices is maximum when their core polarities are opposite ${ }^{13}$, although, higher interaction strength is not the sufficient condition for higher transfer efficiency. For higher amplitude input, the vortex motion enters the non-linear regime and vortex core switching occurs; making it impossible to increase and maintain a large amplitude output and a constant phase relation between gyrotropic motion of both the vortices. On the other hand, if the input signal is very weak and the frequency is close to the gyration frequency of the vortex core, the amplitude of the response gradually increases indicating that the core switching may occur at some point, which is not desirable for device application.

In our results we show that off-resonant signals ${ }^{31}$ of lower amplitude can be used to design suitable transducers with isolated vortices, which will be required to convert other kinds of signals (e.g. a rotating field) to vortex core gyration. In the case of a pair of magnetostatically coupled vortices, if a signal is applied to only of them then the other one shows a greater core gyration i.e., amplification when the core polarities are opposite. Antivortex solitons moving through the stray field are held responsible for this behaviour. We postulate some rules regarding their dynamics and use them to mimic transistor-like operations of switching and amplification with a chain of three vortices. Furthermore, we attempt to couple the output of this three vortex chain to two symmetrically placed daughter chains in an attempt to demonstrate a fan-out operation. However, the antivortices involved in the dynamics favoured one branch over the other resulting in a higher level of asymmetry - one of the branches received more power than the other.

\section{Results}

We use permalloy ( $\mathrm{Py}: \mathrm{Ni}_{80} \mathrm{Fe}_{20}$ ) with negligible magneto-crystalline anisotropy in the form of a $40 \mathrm{~nm}$ thin disk of diameter $2 R=200 \mathrm{~nm}$ to ensure a stable vortex structure ${ }^{32}$. The darker shade in Fig. 1 (a) represents such an isolated vortex. Figure 1 (b) shows a pair of coupled vortices whose centre to centre distance is $a=250 \mathrm{~nm}$. A chain of three vortices, with the same centre to centre distance $a$, has also been studied with different orientations of polarity. Spatially averaged $X$-component of magnetization $\left\langle m_{x}\right\rangle$ has been used as an indicator of core displacement away from their equilibrium positions. The square of the amplitude of Fourier transform of $\left\langle m_{x}\right\rangle(t)$ (with respect to $t$ ) - also known as the energy spectral density (ESD) shows the peaks in vortex core dynamics as a function of frequency.

Isolated magnetic vortex. Figure 1 (c) shows a plot of $\left\langle m_{x}\right\rangle$ vs. time and Fig. 1 (d) shows the associated ESD (in decibel) for the single vortex excited by a broadband signal. The gyrotropic mode is observed at frequency $f=f_{0} \approx 1.27 \mathrm{GHz}$. Higher frequency modes associated with the generation of spin-waves ${ }^{33}$ are also observed. Here, we concern ourselves with frequencies $f \leq f_{0}$ while using signals that are rotating CW or CCW in the plane of the magnetic vortices. With up polarity $p=1$, CCW signals are known to produce greater gyration ${ }^{10}$ which can lead to polarity switching. This can be useful in terms of data storage ${ }^{31}$. However, if the polarity switching is somehow avoided, one can use this to create a suitable transducer for appropriately rotating signals. To that end we can use signals with lower amplitude. Signals with off-resonant frequency $f<f_{0}$, should be used to reduce the convergence time. As shown in the Supplementary Fig. F1, anharmonicity of the gyration dynamics results in a beating frequency when off-resonant signals are used. A trade-off between convergence time and beating frequency needs to be further explored as a design consideration.

Coupled magnetic vortices pair. We have examined the transfer of energy from one vortex to another in terms of their core gyration amplitude (measured in terms of $\left\langle m_{x}\right\rangle$ ) when excitation is only given
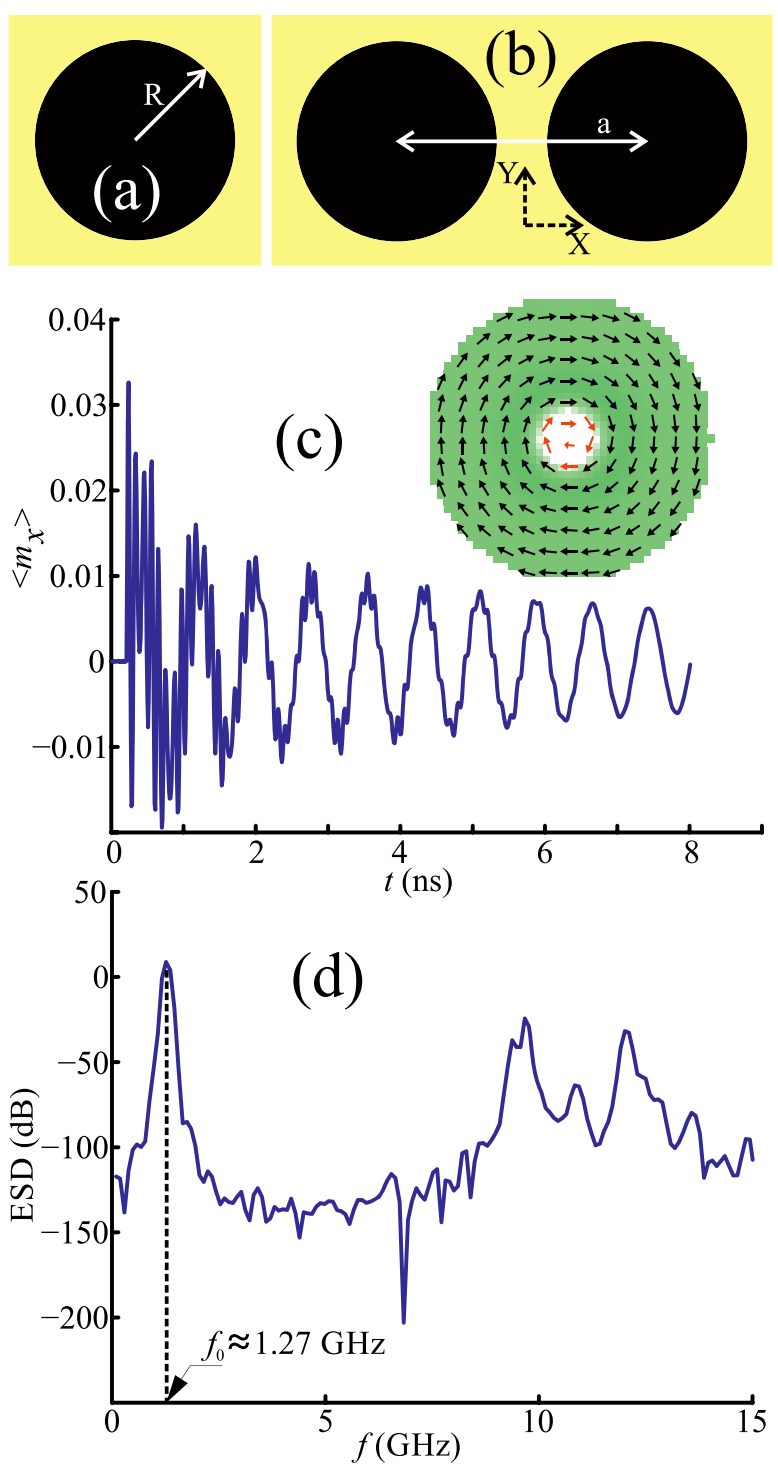

Figure $1 \mid$ Isolated vortex and coupled vortex pair. Dark regions represent the $40 \mathrm{~nm}$ thick (a) isolated and (b) coupled magnetic vortices each of diameter $2 R=200 \mathrm{~nm}$. The centre to centre separation in the case of coupled vortices is set to $a=250 \mathrm{~nm}$. (c) Time evolution and (d) corresponding energy spectral density of $\left\langle m_{x}\right\rangle$ in response to the signal $H_{x}^{S}$.

to one of them. Here, $\left\langle m_{x}\right\rangle$ is computed for both the vortices separately. The dynamics was examined with all sixteen combinations of polarity and chirality of the two vortices. When a small external bias field is applied along the $\mathrm{X}$-axis, the vortex cores may move up or down along the $Y$-axis. This changes their separation and causes magnetic surface charges to appear on the vortex boundaries; consequently affecting the strength of their magnetostatic coupling ${ }^{34,35}$. Hence, in the presence of a bias field, if both the vortices have the same chirality, their coupling will remain relatively unaffected, than when they have different chiralities. This phenomena can be used to affect a chirality dependent dynamics and signal transmission. However, in the absence of an external bias, we observed that chirality does not play any role towards enhancing the asymmetry in dynamics, as described below. Thus, henceforth all vortices in this work have CCW chirality. Furthermore, observable changes only appeared to occur between cases with similar and opposite polarities $^{14}$. Mediated by several factors ${ }^{8,36-39}$, the resonant frequencies of a pair of vortices can differ from that of an isolated vortex. However, in this study, we used an excitation signal rotating 


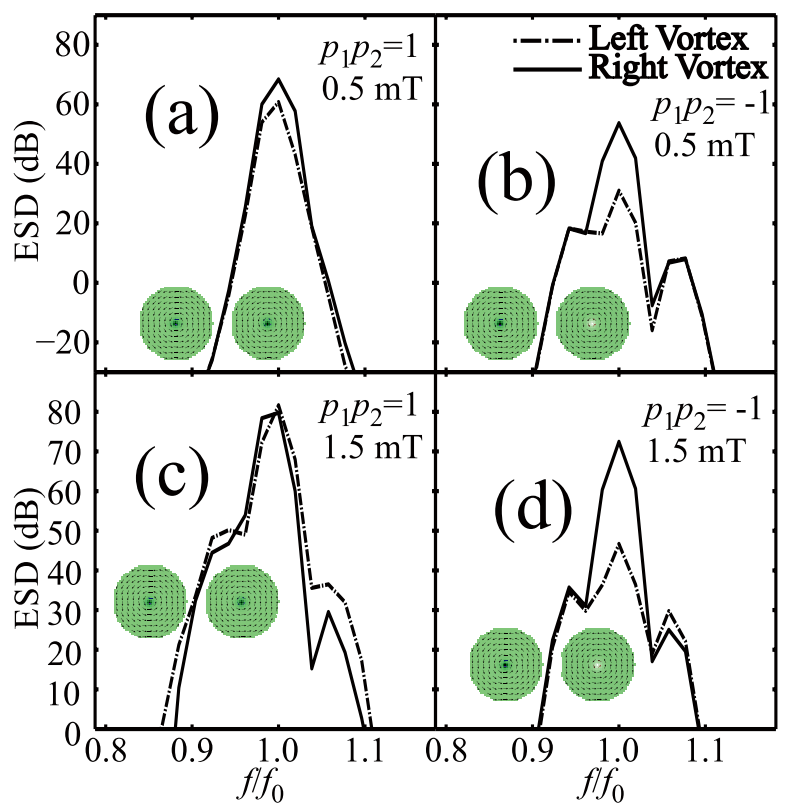

Figure $2 \mid$ Gyration amplification. ESDs of left and right magnetic vortices shown in the insets with ((a) and (c)) similar and ((b) and (d)) opposite polarities. (a) and (b) show the results for a signal amplitude of $0.5 \mathrm{mT}$ while (c) and (d) show those for an amplitude of $1.5 \mathrm{mT}$, both rotating CCW at $f=f_{0}$.

at frequency $f_{0}$, which is applied only on the left disk (Fig. 1 (b)). Figures 2 (a) and (c) show the results for the case when both polarities are up $\left(p_{1} p_{2}=1\right)$ and Figs. $2(\mathrm{~b})$ and $(\mathrm{d})$ show those when left core is up and right core is down $\left(p_{1} p_{2}=-1\right)$. Figures 2 (a) and (b) correspond to a signal amplitude of $0.5 \mathrm{mT}$ and Figs. 2 (c) and (d) correspond to a signal amplitude of $1.5 \mathrm{mT}$.

With increase in the signal amplitude from $0.5 \mathrm{mT}$ to $1.5 \mathrm{mT}$, we see a splitting ${ }^{40}$ of the peak. Furthermore, when $p_{1} p_{2}=-1$, more energy is transmitted and stored in the right vortex. For a signal amplitude of $0.5 \mathrm{mT}$ and $p_{1} p_{2}=1$, the left vortex, exhibits $60.72 \mathrm{~dB}$ of ESD at its gyrotropic mode while the right vortex exhibits $68.43 \mathrm{~dB}$. While for $p_{1} p_{2}=-1$, these values become $30.99 \mathrm{~dB}$ and $53.69 \mathrm{~dB}$, respectively. Thus, the difference in ESDs of gyrotropic modes of left and right vortices increases by about $15 \mathrm{~dB}$. When the signal amplitude is increased to $1.5 \mathrm{mT}$, these values become $81.66 \mathrm{~dB}$ (left vortex) and $79.76 \mathrm{~dB}$ (right vortex) for $p_{1} p_{2}=1$ and $46.67 \mathrm{~dB}$ (left vortex) and $72.45 \mathrm{~dB}$ (right vortex) for $p_{1} p_{2}=-1$. Here (for $p_{1} p_{2}=-1$ ), the ESD (at $f=f_{0}$ ) of the right vortex is $25.78 \mathrm{~dB}$ greater than that of the left vortex. This shows that opposite core polarity facilitates amplification of signal transfer, which is further enhanced by the input signal amplitude. An increase in about $9.5 \mathrm{~dB}$ in signal power (from $0.5 \mathrm{mT}$ to $1.5 \mathrm{mT}$ ) has caused the difference in gain to increase by $12.69 \mathrm{~dB}$. The dependence of this relative amplification on the strength of the input signal is essential to mimic the transistor operation where the base current controls the amplification.

These observations, along with the ones made for an isolated vortex, testify to the existence of anharmonic and asymmetric dynamics present in the vortex core gyration, which cannot be explained by solutions of the Thiele's equation with linear approximations ${ }^{23,41,42}$; even if vortex core deformation ${ }^{43}$ is taken into account. Although, the type of amplification described here has not been seen before, asymmetry based on polarity in terms of energy transfer rate and efficiency has been observed experimentally. Stronger or weaker stray field coupling can affect the rate of energy transmission ${ }^{13}$, but it does not guarantee the observed asymmetry in general and amplification in particular.

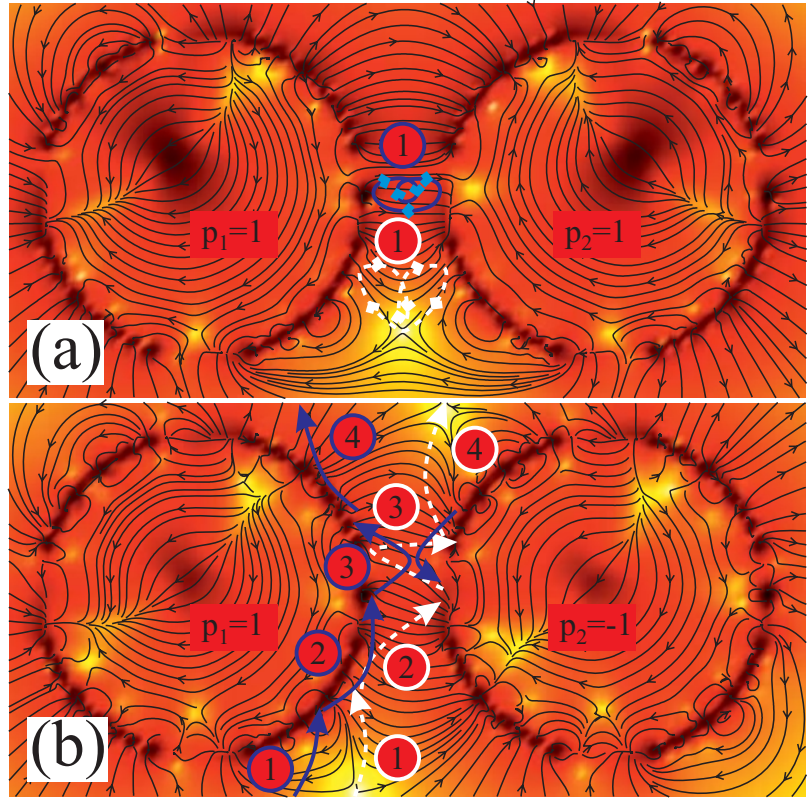

Figure $3 \mid$ Stray field distribution showing the path of travelling antivortex packets for $p_{1} p_{2}=1$ (a) and $p_{1} p_{2}=-1(\mathrm{~b})$. With time the packets shift their path from the dashed to the solid lines.

One may draw an analogy of this observation with a driven double pendulum made of identical pendulums. In this case, when the driving frequency is same as the eigenfrequency of the isolated pendulum then the top pendulum mass becomes stationary while the bottom pendulum mass moves with an amplitude twice as that of the driving amplitude. This may be considered as an infinite amplification (although, the angular displacement of the top pendulum will still not be zero). However, for a coupled pendulum, where two pendulums are connected with a spring and dynamics is not pinned at any end (this system is closer to the coupled vortices presented here in terms of the underlying equations of their dynamics), no such amplification occurs when the driving frequency is the same as the eigenfrequency of the individual pendulum ${ }^{44}$ (see Supplementary Note N1). Either way, a direct comparison of the energy transfer mechanism of coupled magnetic vortices with that of coupled mechanical oscillators is difficult due to the presence of additional parameters in the former case. The gyrovector, which serves as the inertia of the vortex core ${ }^{7}$ can switch direction with core polarity, resulting in the amplification observed here. Hypothetically, this will be comparable to obtaining an amplification in one of the coupled pendulums by changing its inertia from $I$ to $-I$. An analytical model is yet to be developed to describe this phenomenon; but, this is outside the scope of this paper (see Supplementary Note N1). Here, we considered a numerical approach and calculated the temporal evolution of the stray field and discovered that packets of antivortex structures travelling through the stray field mediate the transfer of energy between the two vortices. The path of these antivortices are shown in Fig. 3 for both polarity combinations when the excitation signal amplitude is at $1.5 \mathrm{mT}$. As time progresses the path shifts from dashed to solid lines.

As seen from Fig. 3 (a), a single antivortex packet moves in a closed path for $p_{1} p_{2}=1$. This packet collides with other antivortex structures which originate at the boundaries of the nanodisks. We understand that the antivortex packets discussed here are not particles in the true sense and their apparent 'collision' is only a result of the interacting stray fields. This interaction (or collision) is also shown in the Supplementary Movie M1. It is only during this collision that these antivortex 'solitons' ('soliton' has been used loosely here to describe even those short-lived antivortex packets which do not 
possess consistent form for significant duration) are allowed to change their size (local field distribution) significantly ${ }^{45}$. When it collides with the left disk, which initially has more gyrotropic energy, it becomes smaller and when it collides with the right disk, it becomes larger. This indicates that there is an inverse relation between the size of the antivortex and the gyrotropic energy that it can transfer. As time progresses, and the amplitudes of the gyrating vortex cores become comparable, the path of this bouncing soliton becomes smaller and it moves to a location shown by the solid line (1) in Fig. 3 (a). The soliton itself does not change greatly in size after this point indicating no significant transfer of energy.

Figure 3 (b) shows that more than one antivortex solitons are involved in the energy transfer for the case when $p_{1} p_{2}=-1$. On a given vortex boundary, as one soliton gets terminated, another one is created. This creates a cascade of solitons, which vary in size (see Supplementary Movie M2). At first, the dashed lines mark the path of this cascade with the large arrowheads showing where a soliton gets localized. Branches are numbered from (1) to (4), in an order such that the path of the new soliton is shown by the next branch. The path of this cascade changes gradually with time as well. Specifically, the number of rebounds between the boundaries of the vortices (the length of branch (3) may vary quite often. However, it is observed that as branch (1) terminates, the remaining solitons, which follow branches (2) and (3) are relatively smaller. When branch (3) terminates, a soliton of the same size as the first one (which traversed branch (1) emerges from the right vortex to trace the final branch (4). Thus the right vortex gains gyrotropic energy in the beginning of the dynamics. This cascade occurs twice every cycle, suggesting that signal transfer rate or efficiency can be controlled not only by the saturation magnetization ${ }^{13}$, but also by the frequency of the signal; and further optimization of signal transfer efficiency by tuning the dimensions of the coupled vortices is possible. As time progresses, the cascade starts to occur along the solid lines (1) to (4), shown in Fig. 3 (b). When the gyration amplitude of the right vortex becomes a certain degree greater than that of the left one, we notice that the soliton, which was traversing the dashed branch (3) earlier, now starts from the boundary of the left vortex. However, it is deflected back by another soliton, which emerges from the right vortex - much like an electron or hole charge carrier being prohibited from crossing the depletion layer of a junction diode. We can turn this amplification 'on' or 'off simply by switching the polarity $p_{2}$; but it may be technologically more desirable to have this control via a third vortex.

Magnetic vortex transistor (MVT). In order to examine this transistor-like behaviour, we now add another vortex towards the right of the vortex pair shown in Fig. 1 (b) to form a three vortex sequence with polarities (from left to right) $p_{1}, p_{2}$ and $p_{3}$, which take values of 1 or -1 denoting up or down polarities. In the previous subsection, we identified relative polarity as the source of the observed amplification. Hence, here we study only the four cases with $p_{1}=1$ (up), $p_{2}= \pm 1$ and $p_{3}= \pm 1$. Chirality in all cases is CCW. Signal is applied to the left vortex only. The ESDs for these cases around frequency $f_{0}$ are shown in Fig. 4 (as shown in the insets, the excitation is given to shaded vortices only).

Splitting can be observed in a few cases in Fig. 4. Unlike the splitting seen with increase in signal amplitude (see Fig. 2 (c)), which happens due to inherent non-linearities of the dynamics ${ }^{40}$, here it occurs for a different reason: an increase in the number of vortices leading to an increase in the number of permutations of couplings in the system ${ }^{46}$. Below, we consider any difference in ESD at the signal driving frequency of $f=f_{0}$ only.

As seen in Figs. 4 (a) and (c), the transmission efficiency is equivalent for a persistent signal in both cases: $(1,1,1)$ and $(1,-1,1)$; with the latter faring slightly better. Although, a third vortex was added in the chain, a gain of $12.84 \mathrm{~dB}$ (between right and left most vortices) is observed in Fig. 4 (d). Also, transistor like switching is observed
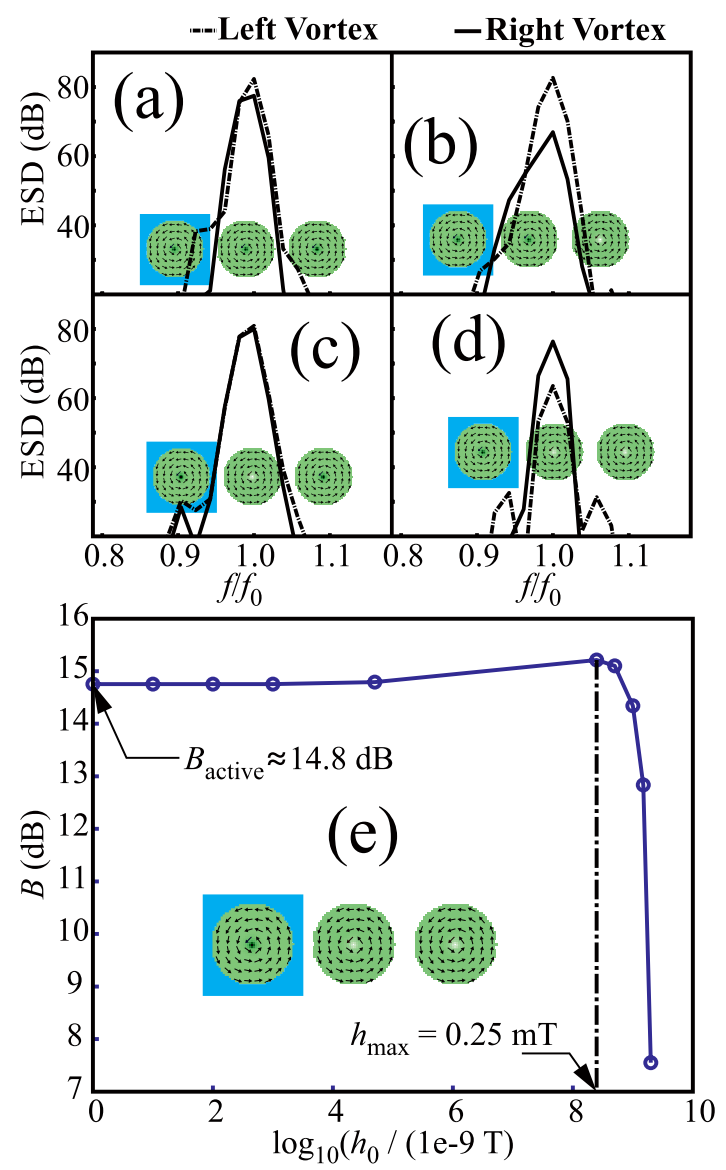

Figure 4 Magnetic vortex transistor. ESDs of left and right magnetic vortices with $\left(p_{1}, p_{2}, p_{3}\right)$ equalling $(\mathrm{a})(1,1,1)$, (b) $(1,1,-1),(\mathrm{c})(1,-1,1)$ and $(\mathrm{d})(1,-1,-1)$ as shown in the respective insets. A $1.5 \mathrm{mT}$ signal rotating CCW at frequency $f_{0}$ is applied only to the left (shaded) vortex. (e) Gain $B$, versus logarithm of signal amplitude $h_{0}$.

clearly with the three vortex sequence considered here when changing from $p_{2}=-1$ (high base current) (Fig. 4 (d)) to $p_{2}=1$ (low base current) (Fig. 4 (b)) changes the difference in signal levels of the right vortex (collector) from $12.84 \mathrm{~dB}$ to $-15.71 \mathrm{~dB}$. We define the gain $B$ in Eq. 1 as below:

$$
B \equiv \operatorname{ESD}_{3}\left(f_{0}\right)-\mathrm{ESD}_{1}\left(f_{0}\right)
$$

Here $\operatorname{ESD}_{1}\left(f_{0}\right)$ and $\operatorname{ESD}_{3}\left(f_{0}\right)$ are $\operatorname{ESD}$ at $f=f_{0}$ for left and right vortices, respectively.

We further checked if this gain $B$, also depended upon the input signal amplitude $h_{0}$. Figure 4 (e) shows a plot of $B$ versus $h_{0}$ for $h_{0}=$ (1e-6, 1e-5, 1e-4, 1e-3, 5e-4, 0.25, 0.5, 1, 1.5, 2) mT. Left vortex's core reversed for $h_{0}=3 \mathrm{mT}$; and hence, we limit ourselves to $2 \mathrm{mT}$. For lower values of $h_{0}$, the gain appears to be constant at $B=B_{\text {active }} \approx$ $14.8 \mathrm{~dB}$. This is reminiscent of a BJT operating under small-signal conditions ${ }^{47}$. At higher signal strength, the gain $B$, no longer remains constant. This indicates that like other electronic transistors, our 'magnetic vortex transistor' is also susceptible to non-linear distortion. The maximum value of gain $B=B_{\max } \approx 15.21 \mathrm{~dB}$ is seen for a signal strength of $h_{0}=h_{\max }=0.25 \mathrm{mT}$.

We further investigate the roles of the stray field antivortex solitons on the transistor-like operations described above. We begin by analysing the temporal evolution of the stray field for cases where $\left(p_{1}\right.$, $\left.p_{2}, p_{3}\right)$ equals $(1,-1,1)$ and $(1,-1,-1)$. The same is shown in Supplementary Movies M3 and M4, respectively. Figures 5 (a) and (b) summarize the path of the solitons involved. Polarity dependent transient gyrotropic energy transfer between any two neighbouring 


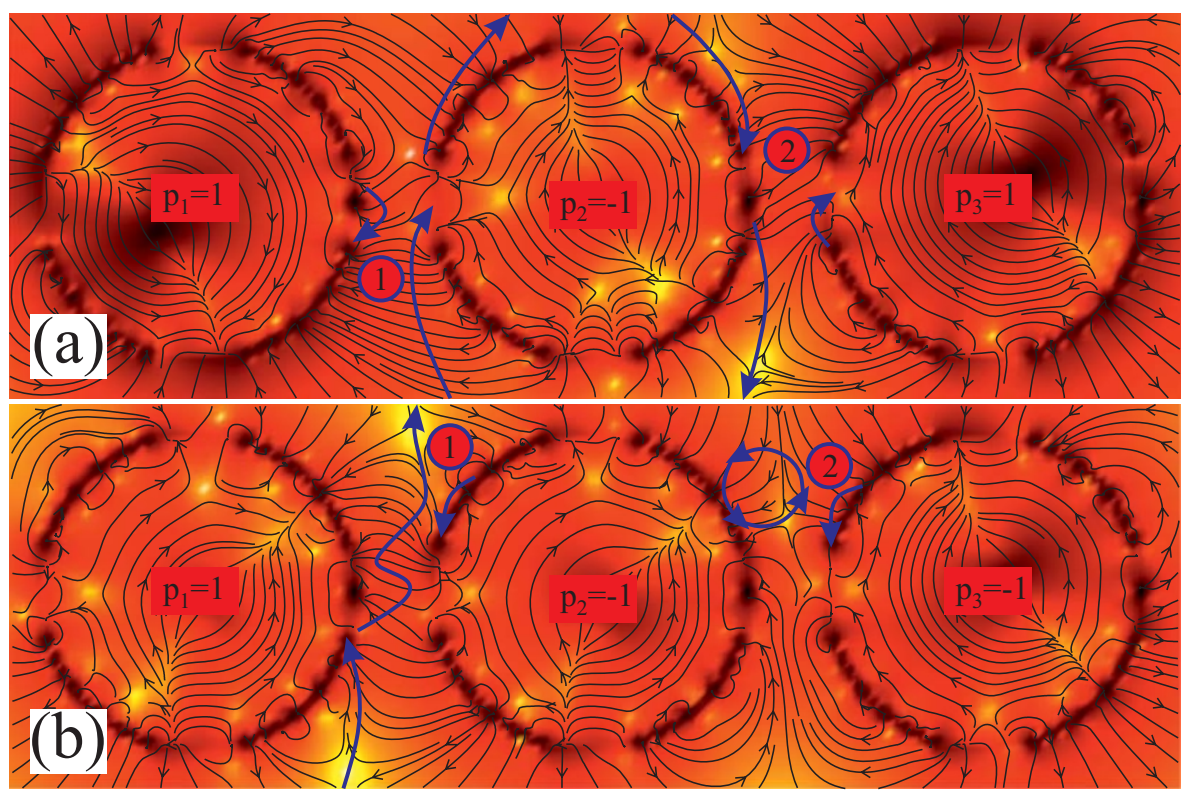

Figure $5 \mid$ Vortex chain. Stray field distribution in the cases where $\left(p_{1}, p_{2}, p_{3}\right)$ equals $(\mathrm{a})(1,-1,1)$ and $(\mathrm{b})(1,-1,-1)$. The path of antivortex packets after the dynamics has stabilized is marked with solid lines.

vortices here too occur in the same manner as shown by the dashed lines in Fig. 3. These lines are omitted in Fig. 5 for clarity. Solid lines show the approximate paths that the solitons follow after the dynamics had become relatively stabilized. For $\left(p_{1}, p_{2}, p_{3}\right)=(1$, $-1,1)$, where no amplification $(B<0 \mathrm{~dB})$ is observed, the cascade of solitons form a large oval loop around the central vortex. Energy appears to be transferred during collisions at $(1$ and (2). This creates a closed feedback loop directly between the left and the right vortices. The solitons skip along the boundary of the central vortex on several occasions in order to aid their own cascade. Most importantly, we note here as well that solitons in the bottom half of the loop (right to left vortex) appear larger (lesser energy) than those traversing the top half (left to right vortex). Thus the flow of energy still occurs from the left vortex, which is excited externally, to the right one. However, an amplification $(B>0 \mathrm{~dB})$ is observed for $\left(p_{1}, p_{2}, p_{3}\right)=(1,-1,-1)$ and Fig. 5 (b) sheds some light on this crucial finding. Here, the path of antivortex solitons between the left and the central vortex does not change greatly from its early transient stage. Here too, energy is transferred at the sites (1) and (2), essentially from the left vortex to the right one. However, unlike in Fig. 5 (a), no feedback loop, and thus no energy rebalancing is present here. This leads to a unidirectional flow of energy as determined by the cascade of antivortex solitons. The right vortex core, thus builds up gyrotropic energy until its drag (or dissipation) matches the power influx. Thus an amplification of the gyrotropic mode of the right vortex is observed in this case. One can simplify the dynamics for the two cases analysed above by considering the central vortex as an efficient medium and taking it out of the picture. Then we can see that amplification was observed when $p_{1} p_{3}=-1$. However this amplification can be controlled by switching the polarity of the middle vortex $\left(p_{2}\right)$, similar to what is done by switching the base current in a BJT.

Fan-out. In an attempt to demonstrate a fan-out operation, which may support the development of more complex circuits, we placed two more MVTs symmetrically above and below the original MVT as shown in Fig. 6. Same material and structural parameters as before were used here. Power was given only to the left vortex of the original MVT. To our surprise, amplification was seen in only one of the branches. As shown in the inset of Fig. 6, the right vortex of upper branch in this network received about $15 \mathrm{~dB}$ more power than the lower branch. When all the core polarities in this network were reversed, the lower branch received the greater power by the same amount.

The cause of this asymmetry is the fact that the solitons do not split during a fan-out. Also, the antivortex seen between the first two vortices in Fig. 5 (b) goes on directly to hit the upper chain as marked in Fig. 6. Thus, further study regarding the implementation of a fanout is warranted by looking at the path of these antivortices in different network configurations.

\section{Discussion}

We numerically examined the polarity dependent asymmetry and non-linearities in vortex dynamics. Cases presented in this paper included isolated vortices and coupled two and three vortex sequences. We particularly examined the dynamics for gain in the transfer of gyrotropic mode power from one vortex to another. To start with, we describe the design considerations in creating a transducer which can convert power from a rotating field signal to gyrotropic power. Best results were observed when the driving signal frequency was close to the eigenfrequency of the transducer. In the case of coupled pair of vortices, when an excitation signal is applied to only one of the vortices then considerably more energy (a maximum gain of $27.68 \mathrm{~dB}$ ) is transferred and stored in its neighbouring vortex if it has the opposite polarity. We further observed that this amplification of energy transfer can be extended over three vortices for a particular case of $\left(p_{1}, p_{2}, p_{3}\right)=(1,-1,-1)$. We interpreted these remarkable observations using the temporal evolution of stray magnetic field and observed that antivortex packets moving through the stray field were accountable for the observed amplifications. The rules, which we postulated based upon the motion of the antivortex packets (or 'solitons') can also successfully explain the previous experimental observations in greater detail. We hope that further study of these solitons will aid the research community in creating a better analytical model which can predict such useful results as signal amplification without the need to do complete simulations.

Similar amplification may be observed in coupled mechanical oscillators. However, here the observed amplification in the energy transfer from left (input) to right (output) vortex for $\left(p_{1}, p_{2}, p_{3}\right)=(1$, $-1,-1$ ) can be controlled by switching the polarity $p_{2}$ from 1 to -1 , much like changing the states of a BJT between active and cut-off. 


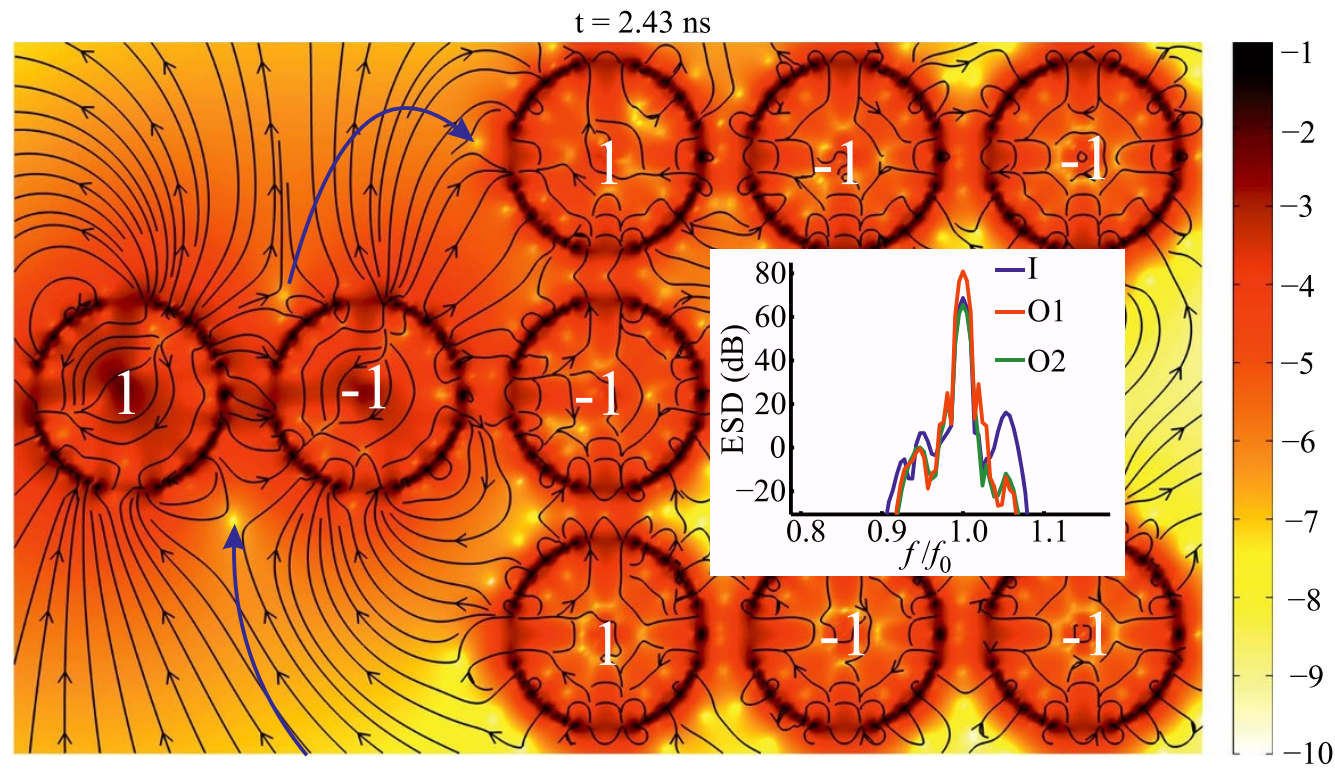

Figure $6 \mid$ Fan-out. Stray field distribution in a MVT network. Signal is given only to the left vortex in the middle row. Vortices are marked with their respective polarities. A path followed by the antivortex packets taking energy from the left vortex in the middle chain to the left vortex of the top chain is marked. The ESDs of left and upper and lower right vortices are given in the inset marked as 'I', 'O1' and 'O2' respectively.

This can be achieved by using a local magnetic field or a spin-polarized current; thus, making it a more suitable candidate for integration with current electronic technological ecosystem. Moreover the observed gain, while remaining constant at $B_{\text {active }}$ for low signal strength $h_{0}$, drops dramatically for $h_{0}>0.25 \mathrm{mT}$. Thus the output will not increase over a certain upper limit $(76.61 \mathrm{~dB}$ for the MVT described here). This is similar to the saturation state of an electronic transistor. Direct parallels to all three operational states of a BJT, namely active, cut-off and saturation have thus been demonstrated for the discussed MVT. Also, both classic transistor operations of signal switching and amplification have been described. The dependence of gain characteristic, in particular $B_{\text {active }}$ and $B_{\max }$ should be further explored with different material and geometrical parameters of the MVT and the driving signal frequency (dynamic response).

Our attempt to demonstrate a fan-out operation uncovered that the solitons involved in the dynamics do not split easily. This resulted in a higher level of asymmetry between different branches of a symmetrical network. This asymmetry was unlikely if the dynamic stray field lacked any topologically stable antivortices and treated both the branches evenly. This helped us further to validate the importance of antivortex solitons in the energy transfer mechanism. More work will be needed to demonstrate a successful fan-out operation by considering the cascade of antivortices for different network parameters.

Pinning, which can occur due to fabrication related issues, is known to affect the natural frequency of an isolated vortex ${ }^{48}$. However, as the dynamics studied here was forced, the observed results are expected to remain unaffected unless the pinning potential is high enough to change the trajectory of the vortex core or the cascade of solitons. A stronger pinning may sometimes occur at the Py-air boundary ${ }^{49}$. We have not considered this type of pinning here as it can affect the generation and dynamics of the stray field which is responsible for some of the reported observations. Thus, a different soft-ferromagnet may have to be used if pinning becomes an issue.

While advancing the cause of nano-electronic devices ${ }^{50}$, we also hope that these findings will promote the continued search of new and improved transistors ${ }^{51}$. For the type of transistor proposed here to become technologically viable and competitive, further research towards miniaturization and reduction of energy consumption and response delay are highly desirable.

\section{Methods}

Magnetic vortex dynamics was simulated using the finite difference method based Landau-Lifshitz-Gilbert (LLG) ordinary differential equation solver called Object Oriented Micro-magnetic Framework (OOMMF). Before the dynamics could be observed, a magnetic ground state has to be achieved with required vortex core polarity and chirality ${ }^{1,2,6,52-57}$. This was accomplished by using a pulse field $H_{t}=H_{0}$ $\exp \left(-t^{\prime 2}\right)$. Here, $\mu_{0} H_{0}=1 \mathrm{~T}$ and normalized time $t^{\prime}=\left(t-t_{0}\right) /(\sqrt{2} \sigma) \cdot t_{0}=75 \mathrm{ps}$ and $\sigma$ is the standard deviation of this Gaussian pulse in time whose full width at half maximum is $30 \mathrm{ps}$. Close to the centre of the circular geometries we apply $H_{z}= \pm H_{t}$ / 10 along the $Z$ axis where the sign controls the core's polarity. If the origin of coordinates is brought to the centre of the vortex then $X$ - and $Y$-components of fields, $H_{x}$ and $H_{y}$ that would produce the desired chirality are given below

$$
\begin{aligned}
& H_{x}=\mp H_{t} \sin (\theta) ; \\
& H_{y}= \pm H_{t} \cos (\theta) .
\end{aligned}
$$

Here, $\theta=\tan ^{-1}(y / x)$ and the upper or lower signs were chosen for CCW or CW chiralities, respectively. It is to be noted that this pulse signal controlled by $H_{t}$, dies down quickly while the magnetic ground state is obtained by running the simulation for $40 \mathrm{~ns}$ under a high damping (Gilbert damping constant $\alpha=0.95$ is used in the LLG equation). We have used saturation magnetization, $M_{s}=0.8 \times 10^{6} \mathrm{~A} / \mathrm{m}$, exchange constant, $A=13 \times 10^{-12} \mathrm{~J} / \mathrm{m}$ and zero magneto-crystalline anisotropy. During vortex dynamics simulations we reduce $\alpha$ to a more realistic value of 0.008 for Py. Magnetization was observed every 10 ps for about 40 ns during dynamics. The cell size used during simulation was $5 \mathrm{~nm} \times 5 \mathrm{~nm} \times 40 \mathrm{~nm}$. To ascertain that the results discussed here are independent of cell size, Fig. 2 was reproduced using a cell size of $2.5 \mathrm{~nm} \times 2.5 \mathrm{~nm} \times 40 \mathrm{~nm}$. The same has been shared as Supplementary Fig. F2. Although the form of the peaks have changed to some extent, the relative gains at $f=f_{0}$ remain largely unaffected.

Before we start to explore the dynamics of magnetic vortices, we first need to obtain the natural frequencies associated with a single isolated vortex. A broadband excitation signal was given to reveal these frequencies. The signal had only $X$-component, $H_{x}^{S}$ which contained power up to $f_{c u t}=45 \mathrm{GHz}$ and depended upon time $t$ as given by:

$$
H_{x}^{S}=H_{x}^{0} \frac{\sin \left(2 \pi f_{c u t}\left(t-t_{0}\right)\right)}{2 \pi f_{\text {cut }}\left(t-t_{0}\right)} .
$$

Here, $\mu_{0} H_{x}^{0}=0.05 \mathrm{~T}$ and $t_{0}=200 \mathrm{ps}$

Upon obtaining the magnetization data from OOMMF, we chose to analyse the results by looking at the time evolution of spatial average of normalized $X$-component of magnetization, $\left\langle m_{x}\right\rangle$ for each vortex, and its corresponding ESD. Normalization is done by dividing the $X$-component of magnetization, $M_{x}$ by $M_{s}$; such that $m_{x}=M_{x} /$ $M_{s}$. The Hanning window is used on $\left\langle m_{x}\right\rangle$ to reduce spectral leakage. The widowed data is then zero padded and Fourier transformed to obtain the required ESD, 
$\left\langle\bar{m}_{x}\right\rangle^{258}$. This is plotted in figures on decibel scale as $w_{H} \times 20 \log _{10}\left|\bar{m}_{x}\right|$, where a window scaling factor of $w_{H}=2$ is used for the Hanning window. These ESDs were calculated after running the dynamics for over $40 \mathrm{~ns}$, so that any transient vortex core dynamics are suppressed and steady state dynamic solutions appear to be more prominent in the spectrum. Power spectral density is considered to be more desirable in the case of persistent signals. However, here we run the simulations for finite amount of time. Also, natural damping ensures that net power input to the system becomes zero before the simulations finish. The stray field is also obtained from OOMMF during dynamics. The stray field plots shown in Figs. 3 and 5 and the supplementary movies were created using MATLAB. The contour colouring is based on the sum of squares of $X$ - and $Y$-components of the stray field.

1. Van Waeyenberge, B. et al. Magnetic vortex core reversal by excitation with short bursts of an alternating field. Nature 444, 461-464; DOI:10.1038/nature05240 (2006).

2. Yamada, K. et al. Electrical switching of the vortex core in a magnetic disk. Nat. Mater. 6, 270-273; DOI:10.1038/nmat1867 (2007).

3. Cowburn, R. P. Spintronics: Change of direction. Nat. Mater. 6, 255-256; DOI:10.1038/nmat1877 (2007).

4. Thomas, J. Magnetism: Vortex ups and downs. Nat. Nanotech. 2, 206-206; DOI:10.1038/nnano.2007.92 (2007).

5. Cowburn, R. Magnetic nanodots for device applications. J. Magn. Magn. Mater. 242-245, Part 1, 505-511; DOI:10.1016/S0304-8853(01)01086-1 (2002).

6. Liu, Y., Gliga, S., Hertel, R. \& Schneider, C. M. Current-induced magnetic vortex core switching in a permalloy nanodisk. Appl. Phys. Lett. 91, 112501; DOI:10.1063/1.2780107 (2007).

7. Shibata, J., Shigeto, K. \& Otani, Y. Dynamics of magnetostatically coupled vortices in magnetic nanodisks. Phys. Rev. B 67, 224404; DOI:10.1103/ PhysRevB.67.224404 (2003).

8. Shibata, J. \& Otani, Y. Magnetic vortex dynamics in a two-dimensional square lattice of ferromagnetic nanodisks. Phys. Rev. B 70, 012404; DOI:10.1103/ PhysRevB.70.012404 (2004).

9. Guslienko, K. Y. Low-frequency vortex dynamic susceptibility and relaxation in mesoscopic ferromagnetic dots. Appl. Phys. Lett. 89, 022510; DOI:10.1063/ 1.2221904 (2006).

10. Lee, K.-S. \& Kim, S.-K. Two circular-rotational eigenmodes and their giant resonance asymmetry in vortex gyrotropic motions in soft magnetic nanodots. Phys. Rev. B 78, 014405; DOI:10.1103/PhysRevB.78.014405 (2008).

11. Metlov, K. L. \& Guslienko, K. Y. Stability of magnetic vortex in soft magnetic nano-sized circular cylinder. J. Magn. Magn. Mater. 242-245, Part 2, 1015-1017; DOI:10.1016/S0304-8853(01)01360-9 (2002).

12. Lee, K.-S., Jung, H., Han, D.-S. \& Kim, S.-K. Normal modes of coupled vortex gyration in two spatially separated magnetic nanodisks. J. Appl. Phys. 110, 113903; DOI:10.1063/1.3662923 (2011)

13. Jung, H. et al. Tunable negligible-loss energy transfer between dipolar-coupled magnetic disks by stimulated vortex gyration. Sci. Rep. 1, 59; DOI:10.1038/ srep00059 (2011).

14. Vogel, A. et al. Coupled vortex oscillations in spatially separated permalloy squares. Phys. Rev. Lett. 106, 137201; DOI:10.1103/PhysRevLett.106.137201 (2011).

15. Vogel, A., Drews, A., Weigand, M. \& Meier, G. Direct imaging of phase relation in a pair of coupled vortex oscillators. AIP Advances 2, 042180; DOI:10.1063/ 1.4771683 (2012)

16. Sugimoto, S. et al. Dynamics of coupled vortices in a pair of ferromagnetic disks. Phys. Rev. Lett. 106, 197203; DOI:10.1103/PhysRevLett.106.197203 (2011).

17. Vansteenkiste, A. et al. X-ray imaging of the dynamic magnetic vortex core deformation. Nat. Phys. 5, 332-334; DOI:10.1038/nphys1231 (2009).

18. Curcic, M. et al. Polarization selective magnetic vortex dynamics and core reversal in rotating magnetic fields. Phys. Rev. Lett. 101, 197204; DOI:10.1103/ PhysRevLett.101.197204 (2008)

19. Weigand, M. et al. Vortex core switching by coherent excitation with single in plane magnetic field pulses. Phys. Rev. Lett. 102, 077201; DOI:10.1103/ PhysRevLett.102.077201 (2009).

20. Bolte, M. et al. Time-resolved x-ray microscopy of spin-torque-induced magnetic vortex gyration. Phys. Rev. Lett. 100, 176601; DOI:10.1103/ PhysRevLett.100.176601 (2008).

21. Mesler, B. L., Buchanan, K. S., Im, M.-Y. \& Fischer, P. X-ray imaging of nonlinear resonant gyrotropic magnetic vortex core motion in circular permalloy disks. J. Appl. Phys. 111, 07D311; DOI:10.1063/1.3678448 (2012).

22. Lee, K.-S. \& Kim, S.-K. Gyrotropic linear and nonlinear motions of a magnetic vortex in soft magnetic nanodots. Appl. Phys. Lett. 91, 132511; DOI:10.1063/ 1.2783272 (2007).

23. Thiele, A. A. Steady-state motion of magnetic domains. Phys. Rev. Lett. 30, 230-233; DOI:10.1103/PhysRevLett.30.230 (1973).

24. Landau, L. \& Lifshits, E. On the theory of the dispersion of magnetic permeability in ferromagnetic bodies. Ukr. J. Phys. 53, 14 (2008). URL http://ujp.bitp.kiev.ua/ files/30 journals/53/si/53SI06p.pdf.

25. Gilbert, T. A phenomenological theory of damping in ferromagnetic materials. IEEE Trans. Magn. 40, 3443-3449; DOI:10.1109/TMAG.2004.836740 (2004).
26. Krüger, B. et al. Harmonic oscillator model for current- and field-driven magnetic vortices. Phys. Rev. B 76, 224426; DOI:10.1103/PhysRevB.76.224426 (2007).

27. Barman, S., Barman, A. \& Otani, Y. Dynamics of 1-d chains of magnetic vortices in response to local and global excitations. IEEE Trans. Magn. 46, 1342-1345; DOI:10.1109/TMAG.2010.2040587 (2010).

28. Barman, S., Barman, A. \& Otani, Y. Controlled propagation of locally excited vortex dynamics in linear nanomagnet arrays. J. Phys. D: Appl. Phys. 43, 335001; DOI:10.1088/0022-3727/43/33/335001 (2010).

29. Jung, H. et al. Logic operations based on magnetic-vortex-state networks. ACS Nano 6, 3712-3717; DOI:10.1021/nn3000143 (2012).

30. Kim, J.-H., Lee, K.-S., Jung, H., Han, D.-S. \& Kim, S.-K. Information-signaltransfer rate and energy loss in coupled vortex-state networks. Appl. Phys. Lett. 101, 092403; DOI:10.1063/1.4748885 (2012).

31. Kammerer, M. et al. Magnetic vortex core reversal by excitation of spin waves. Nat. Commun. 2, 279; DOI:10.1038/ncomms1277 (2011).

32. Scholz, W. et al. Transition from single-domain to vortex state in soft magnetic cylindrical nanodots. J. Magn. Magn. Mater. 266, 155-163; DOI:10.1016/S03048853(03)00466-9 (2003). Proceedings of the 4th International Conference on Fine Particle Magnetism (ICFPM).

33. Zhu, X., Liu, Z., Metlushko, V., Grütter, P. \& Freeman, M. R. Broadband spin dynamics of the magnetic vortex state: Effect of the pulsed field direction. Phys. Rev. B 71, 180408; DOI:10.1103/PhysRevB.71.180408 (2005).

34. Guslienko, K. Y. et al. Eigenfrequencies of vortex state excitations in magnetic submicron-size disks. J. Appl. Phys. 91, 8037-8039; DOI:10.1063/1.1450816 (2002).

35. Barman, A., Barman, S., Kimura, T., Fukuma, Y. \& Otani, Y. Gyration mode splitting in magnetostatically coupled magnetic vortices in an array. J. Phys. D: Appl. Phys. 43, 422001; DOI:10.1088/0022-3727/43/42/422001 (2010).

36. Buchanan, K. S. et al. Soliton-pair dynamics in patterned ferromagnetic ellipses. Nat. Phys. 1, 172-176; DOI:10.1038/nphys173 (2005).

37. Belanovsky, A. D. et al. Phase locking dynamics of dipolarly coupled vortex-based spin transfer oscillators. Phys. Rev. B 85, 100409; DOI:10.1103/ PhysRevB.85.100409 (2012).

38. Zaspel, C. E. Phase locking of vortex-based spin-torque nanocontact oscillators by antivortices. Appl. Phys. Lett. 102, 052403; DOI:10.1063/1.4790287 (2013).

39. Guslienko, K. Y., Buchanan, K. S., Bader, S. D. \& Novosad, V. Dynamics of coupled vortices in layered magnetic nanodots. Appl. Phys. Lett. 86, 223112; DOI:10.1063/1.1929078 (2005).

40. Buchanan, K. S., Grimsditch, M., Fradin, F. Y., Bader, S. D. \& Novosad, V. Driven dynamic mode splitting of the magnetic vortex translational resonance. Phys. Rev. Lett. 99, 267201; DOI:10.1103/PhysRevLett.99.267201 (2007).

41. Thiele, A. A. Applications of the gyrocoupling vector and dissipation dyadic in the dynamics of magnetic domains. J. Appl. Phys. 45, 377-393; DOI:10.1063/ 1.1662989 (1974).

42. Huber, D. L. Dynamics of spin vortices in two-dimensional planar magnets. Phys. Rev. B 26, 3758-3765; DOI:10.1103/PhysRevB.26.3758 (1982).

43. Ha, J. K., Hertel, R. \& Kirschner, J. Micromagnetic study of magnetic configurations in submicron permalloy disks. Phys. Rev. B 67, 224432; DOI:10.1103/PhysRevB.67.224432 (2003).

44. Bajaj, N. K. The Physics of Waves and Oscillations (Tata McGraw - Hill, 1988).

45. Kanna, T. \& Lakshmanan, M. Exact soliton solutions, shape changing collisions, and partially coherent solitons in coupled nonlinear schrödinger equations. Phys. Rev. Lett. 86, 5043-5046; DOI:10.1103/PhysRevLett.86.5043 (2001).

46. Han, D.-S. et al. Wave modes of collective vortex gyration in dipolar-coupled-dotarray magnonic crystals. Sci. Rep. 3, 2262; DOI:10.1038/srep02262 (2013).

47. Bogart, T. F. Electronic Devices and Circuits, chap. 5, 134-195 (Universal Book Stall, New Delhi, New Delhi, 2000).

48. Compton, R. L. \& Crowell, P. A. Dynamics of a pinned magnetic vortex. Phys. Rev. Lett. 97, 137202; DOI:10.1103/PhysRevLett.97.137202 (2006).

49. Kłos, J. W. et al. Effect of magnetization pinning on the spectrum of spin waves in magnonic antidot waveguides. Phys. Rev. B 86, 184433; DOI:10.1103/ PhysRevB.86.184433 (2012)

50. Klos, J. W., Kumar, D., Krawczyk, M. \& Barman, A. Magnonic band engineering by intrinsic and extrinsic mirror symmetry breaking in antidot spin-wave waveguides. Sci. Rep. 3, 2444; DOI:10.1038/srep02444 (2013).

51. Volz, J. \& Rauschenbeutel, A. Triggering an optical transistor with one photon. Science 341, 725-726; DOI:10.1126/science.1242905 (2013).

52. Yakata, S. et al. Chirality control of magnetic vortex in a square py dot using current-induced oersted field. Appl. Phys. Lett. 99, 242507; DOI:10.1063/ 1.3669410 (2011)

53. Jaafar, M. et al. Control of the chirality and polarity of magnetic vortices in triangular nanodots. Phys. Rev. B 81, 054439; DOI:10.1103/PhysRevB.81.054439 (2010).

54. Pigeau, B. et al. Optimal control of vortex-core polarity by resonant microwave pulses. Nat. Phys. 7, 26-31; DOI:10.1038/nphys1810 (2011).

55. Kikuchi, N. et al. Vertical bistable switching of spin vortex in a circular magnetic dot. J. Appl. Phys. 90, 6548-6549; DOI:10.1063/1.1416132 (2001)

56. Curcic, M. et al. Magnetic vortex core reversal by rotating magnetic fields generated on micrometer length scales. Phys. Status Solidi B 248, 2317-2322; DOI:10.1002/pssb.201147208 (2011).

57. Jain, S. et al. From chaos to selective ordering of vortex cores in interacting mesomagnets. Nat. Commun. 3, 1330; DOI:10.1038/ncomms2331 (2012). 
58. Kumar, D., Dmytriiev, O., Ponraj, S. \& Barman, A. Numerical calculation of spin wave dispersions in magnetic nanostructures. J. Phys. D: Appl. Phys. 45, 015001; DOI:10.1088/0022-3727/45/1/015001 (2012).

\section{Acknowledgments}

We acknowledge the financial support from the Department of Science and Technology, Government of India (grant nos. INT/EC/CMS (24/233552), SR/NM/NS-53/2010 and SR/ WOS-A/PS-27/2010) and Department of Information Technology, Government of India (grant no. 1(7)/2010/M\&C). D.K. would like to acknowledge financial support from CSIR Senior Research Fellowship (File ID: 09/575/(0090)/2011 EMR-I).

\section{Author contributions}

S.B. and A.B. planned the project while D.K. performed the numerical calculations. All authors discussed and interpreted the results, and contributed to the writing of the manuscript.

\section{Additional information}

Supplementary information accompanies this paper at http://www.nature.com/ scientificreports

Competing financial interests: The authors declare no competing financial interests.

How to cite this article: Kumar, D., Barman, S. \& Barman, A. Magnetic Vortex Based Transistor Operations. Sci. Rep. 4, 4108; DOI:10.1038/srep04108 (2014).

(2) $\Theta$ This work is licensed under a Creative Commons Attribution-

NonCommercial-NoDerivs 3.0 Unported license. To view a copy of this license, visit http://creativecommons.org/licenses/by-nc-nd/3.0 